\title{
Antidepressivum verbessert Hirnfunktion nach Schlaganfall
}

\author{
Ein Antidepressivum kann mög- \\ licherweise dazu beitragen, dass \\ Patienten nach einem Schlaganfall \\ kognitive Ausfälle schneller kompen- \\ sieren.
}

- Forscher der University of Iowa, Iowa City, studierten die Effekte des Antidepressivums Escitalopram (ETP), eines selektiven Serotonin-Wiederaufnahmehemmers (SSRI), an 129 Schlaganfall-Patienten in einer randomisierten, doppelblinden, placebokontrollierten Studie.

Innerhalb der ersten drei Monate nach dem Schlaganfall erhielten 43 Patienten über zwölf Monate 5-10 mg ETP täglich. 45 nahmen täglich ein Placebo ein und 41 wurden psychotherapeutisch behandelt. Mit neuropsychologischen Standardtests wurden die ETP-Effekte auf die Ausgangswerte gemessen.

In den drei Tests, mit denen die Gesamtlernfunktion (Denken, Lernen und Gedächtnis) und auf diese aufbauend das verbale und visuelle Gedächtnis gemessen wurde, waren die Differenzen zwischen den untersuchten Gruppen immer signifikant ( $<<0,01$ ). Die Schlaganfallpatienten, die in der Rehaphase ETP bekamen, erlangten dabei ihre geistigen Fähigkeiten schneller und umfassender zurück als die Kontrollen.

\section{Kommentar}

Der positive Effekt des ETP auf die kognitive Erholung ist unabhängig von seinen Wirkungen auf die depressiven Symptome und wird weder vom Typ noch vom Mechanismus des Schlaganfalls beeinflusst.

ETP wurde gut toleriert und Nebenwirkungen waren nicht häufiger als in der Placebogruppe. Die Wirkung des Antidepressivums lässt sich vermutlich mit Strukturveränderungen im Hippocampus und im visuellen und zerebralen Kortex erklären. Die Erholung der Hirnfunktionen nach einem Schlaganfall unter ETP ist so beeindruckend, dass hier unbedingt weiter geforscht werden sollte.

K. MALBERG =

\footnotetext{
- R. E. Jorge et al.

(Dept Psychiatry, Univ of lowa Hospital and Clinics, 200 Hawkins Dr, lowa City, IA 52242 USA) Escitalopram and enhancement of cognitive recovery following stroke. Arch Gen Psychiatry 67 (2010) 187-287
}

\section{Hilft Akupunktur autistischen Kindern?}

Chinesische Forscher randomisierten 30 autistische Kinder in zwei Gruppen. Die Experimentalgruppe erhielt über sechs Wochen 30 Sitzungen mit Akupunktur. Die Kontrollgruppe wurde nicht behandelt. Der Therapieeffekt wurde von den Eltern auf einer eigens entwickelten Bewertungsskala vermerkt. Zusätzlich wurde bei 16 Kindern ein qualitatives EEG herangezogen.

Beide Studienendpunkte lieferten positive Effekte, die für die Wirksamkeit der Akupunktur sprachen. Die Autoren meinen daher, dass diese Therapie bei Autismus effektiv sei.

\section{Kommentar}

Da bei der Hälfte der Kinder eine EEG-Messung nicht möglich war, kommt diesem Parameter wohl kaum
Bedeutung zu. Bleibt also die elterliche Bewertung der Symptomatik. Hier ist zu bemängeln, dass die Skala nicht validiert war. Bedeutend ist ferner, dass es sich hier um eine offene Studie handelt. Es wurde also gar nicht versucht, unspezifische Therapieeffekte zu erfassen. Die beobachteten Resultate müssen also nicht das Geringste mit Akupunktur zu tun haben.

Autismus ist ein Leiden, das die Betroffenen schwer belastet. Man sollte ihnen nicht mit solch fragwürdigen Daten falsche Hoffnungen machen.

E. ERNST =

\section{- A. S. Chanet al.}

Seven-star needle stimulation improves language and social interaction of children with autistic spectrum disorders. Am. J. Chin. Med. 37 (2009) 495-504

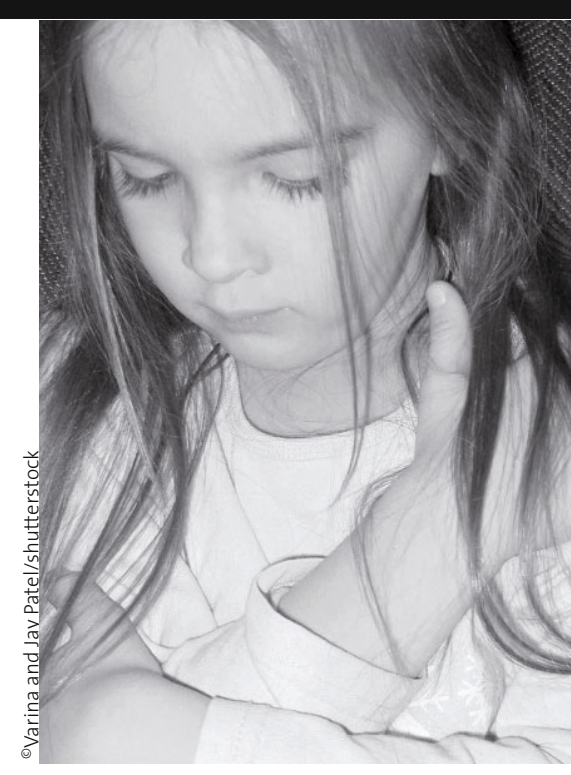

Autistisches Kind: Macht man den Eltern mit fragwürdigen Daten falsche Hoffnungen? 